\title{
EUTOMIA
}

Revista de Literatura e Linguística

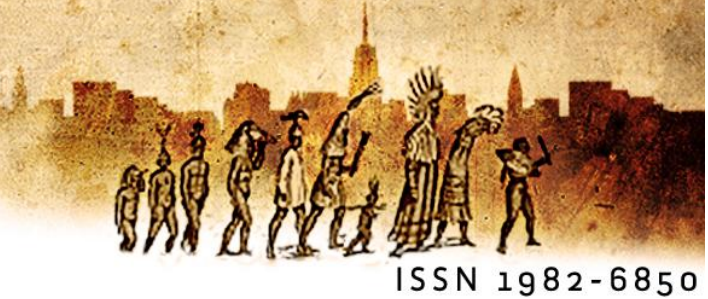

\section{Le problème du dialogue dans les travaux de V. V. Vinogradov (dans les années 1920-1930) $^{1}$}

Irina Ivanova' (Université de Lausanne)

\begin{abstract}
Résumé: Le présent article est consacré à la conception du dialogue chez V. Vinogradov. On suit le développement de sa conception dès ses premiers articles jusqu'à la fin des années 1930, en montrant comment ses idées du dialogue ont été liées à sa théorie de l'analyse des œuvres littéraires. Nous mettons en relief le fait que Vinogradov distinguait trois aspects du dialogue: dialogue comme un procédé de la caractéristique langagière du personnage, dialogue comme un de deux types principaux de la composition et le dialogue comme un moyen d'analyse de la polyphonie de l'œuvre littéraire.
\end{abstract}

Mots-clés: dialogue, dialogisme, monologue, narration, skaz, composition, langage, stylistique, Vinogradov, image d'auteur, œuvre littéraire.

Resumo: Este artigo apresenta a concepção do diálogo em V. Vinogradov. Segue- se o desenvolvimento de sua concepção a partir dos seus primeiros artigos até o fim dos anos 1930, mostrando como as suas ideias do diálogo estavam ligadas a sua teoria de análise das obras literárias. Ressalta-se o fato de que Vinogradov distinguia três aspectos do diálogo: diálogo como um procedimento da característica linguageira do personagem; diálogo como um dos dois principais tipos da composição e o diálogo como um meio de análise da polifonia da obra literária.

Palavras chave: diálogo, dialogismo, monólogo, narração, skaz, composição, linguagem, estilística, Vinogradov, imagem do autor, obra literária.

\footnotetext{
${ }^{1}$ Este artigo foi publicado primeiramente no Cahiers de l'ILSL, N 40,2014 , pp.87-114, 2014 - Université de Lausanne. Sua publicação na Revista Eutomia foi autorizada pela autora. Alguns ajustes no texto foram realizados pela autora, para esta versão A formatação do presente artigo teve que ser modificada para atender às normas da ABNT e da revista. Foi acrescentado também o resumo na língua portuguesa.
} 
Le problème du dialogue et du dialogisme qui s'y rapporte est un des thèmes les plus discutés de la linguistique et de la critique littéraire de la dernière décennie du $X X^{e}$ et de la première décennie du XXI ${ }^{\mathrm{eme}}$ siècle. Des centaines de publications parues au cours de ces vingt années ont étudié ces phénomènes non seulement dans la langue et dans la littérature, mais aussi dans la sociologie, dans la psychologie et dans l'art. Ces études mentionnaient le plus souvent Mikhaill Bakhtine (1895-1975) en tant que fondateur de la théorie du dialogisme, un des premiers philosophes, critique littéraire et linguiste, qui s'est occupé de l'analyse du dialogue. Les discussions autour de Bakhtine et, en particulier, les discussions autour du problème de l'auteur des travaux de Valentin Vološinov (1895-1936) et de Pavel Medvedev (1892-1938), attribués souvent à Bakhtine, ont provoqué de l'intérêt pour le début du $X X^{e}$ siècle, période qui se caractérise par le processus de recherche de nouvelles méthodes dans la critique littéraire et dans la linguistique. Cela a contribué à la parution des études intéressantes sur le formalisme russe, cela a tiré également de l'oubli les noms des linguistes tels que Lev Jakubinsky (1892-1945), Lev Ščerba (1880-1944), Evgueni Polivanov (1891-1938). Ces travaux ont permis de découvrir la richesse de la pensée scientifique et créative de la Russie des années 1920-1930 en brisant le stéréotype occidental de la prégnance de l'idéologie marxiste.

L'analyse du contexte scientifique dans lequel les idées de Bakhtine avaient vu jour fit réfléchir les linguistes sur la question de la comparaison de la conception de Bakhtine avec les idées de ses contemporains. C'est Victor Vladimirovič Vinogradov (1895-1969) qui occupe ici une place de choix. Dans sa monographie consacrée à la situation de la linguistique en Russie au début du $X X^{e}$ siècle (Vološinov, Bakhtine et la linguistique), le célèbre linguiste russe Vladimir Alpatov consacre un chapitre entier à la comparaison de ces deux chercheurs. Il les nomme, à juste titre, «les chercheurs russes les plus importants et les plus connus de leur génération dans le domaine des sciences humaines» (ALPATOV, 2005, pp. 50-56) et il note la complexité, et même un certain côté mystérieux de leur relation. En effet, leurs intérêts scientifiques s'entrecroisaient sous beaucoup de rapports: les problèmes de langue de l'œuvre littéraire, les tâches de la stylistique, l'«image» de l'auteur, le dialogue... Cet entrecroisement déjà été analysé dans les travaux de N. Perlina (1995a, 1995b), d'A. Bolšakov (1996, 1999) et de V. Alpatov (2005).

La comparaison de la conception du dialogisme chez Vinogradov et chez Bakhtine est au centre de l'article de référence de N. Perlina «Le dialogue sur le dialogue» (PERLINA, 
1995b). Pourtant, dans cet article, Vinogradov et Bakhtine sont représentés en «interlocuteurs», c'est-à-dire, l'accent est mis sur la comparaison de leurs points de vue, sur leur «duel», pour parler avec Perlina. En outre, le dialogue en tant que phénomène de langue à proprement parler y est considéré dans ses liens étroits avec l'image de l'auteur, avec le progrès de la stylistique et avec le phénomène qui a reçu le nom de «dialogisme», c'est-à-dire l'aspect communicatif du discours d'auteur. A cause d'une telle approche, la parole dialogale fait partie intégrante de plusieurs phénomènes complexes, elle perd son caractère bien défini et sa spécificité, elle se perd elle-même dans l'analyse littéraire.

Dans l'optique qui est la nôtre, il nous paraît important de mettre au jour les idées de Vinogradov sur le dialogue vu traditionnellement comme conversation entre deux ou plusieurs personnes, c'est-à-dire comme parole des personnages. Pour restaurer la chronologie de ces points de vue, il est indispensable d'analyser les travaux de Vinogradov des années 1920-1930 qui, bien que relatifs à la critique littéraire, s'appuient, néanmoins, sur des recherches des linguistes russes et européens. Cela nous permettra de définir les sources et la spécificité de ses idées sur le dialogue.

\section{De la stylistique du « skaz » au dialogue}

L'intérêt de Vinogradov pour le dialogue est né dans les années 1920, lors des discussions des formalistes russes au sujet du «skaz ». Bien qu'il n'ait pas fait partie de ce courant scientifique de manière officielle, il en fut assez proche à cause de son intérêt pour la poétique de la littérature russe, pour les recherches des méthodes objectives de l'analyse des œuvres de fiction.

En travaillant au Département de la littérature de l'Institut d'Etat de l'histoire des arts [Gosudarstvennyj institut istorii iskusstv - I'abréviation GIII] depuis 1921, il se trouva dans le milieu des formalistes et des philologues proches de ce milieu, tels que V. Žirmunsky (1891-1971), Y. Tynianov (1894-1943), V. Šklovsky (1893-1984), B. Eikhenbaum (1886-1959), B. Engelgardt (1887-1947). En s'intéressant aux études du langage poétique, à la mise en évidence de sa spécificité par rapport au langage pratique, ils s'occupèrent d'abord de l'analyse de la phonétique du langage poétique (les travaux d'O. Brik, de S. Bernštein, de V. Žirmunsky, de B. Eikhenbaum, de V. Šklovsky, de L. Jakubinsky, de R. Jakobson). Ensuite, leur attention porta sur la prose des oeuvres littéraires. 
Un petit article intitulé «L'illusion du skaz» [Illuzia skaza] (1918) de B. Eikhenbaum (1896-1959) devint le premier travail des formalistes russes consacré à l'analyse de la prose. Dans cet article, l'auteur souligne l'évidence de l'opposition de la langue orale à la langue écrite en montrant que l'influence du «skaz» oral peut s'exercer dans la structure des tournures syntaxiques et dans le choix des mots, et même dans la composition d'une oeuvre littéraire. En citant, à titre d'exemple, les oeuvres de Dostoïevsky, de Tourgueniev, de Pouchkine, de Gogol, de Leskov, de Rémizov où le narrateur joue un rôle important, Eikhenbaum, affirme qu'« un vrai créateur de littérature porte ne lui-même des forces primitives, mais organiques de l'art vivant de la narrativité »(EIKHENBAUM, 1924 [1918], p. 156) et il lance un appel aux philologues d'y prêter attention. Ainsi, chez Eikhenbaum, le genre du «skaz» est directement lié à la transmission de la langue orale par écrit.

C'est en 1919 qu'Eikhenbaum publie son célèbre article - l'analyse de la nouvelle de Gogol Le manteau [Šinel'] faite à l'aide de l'approche formaliste, nouvelle pour l'époque. II y prête une attention particulière aux moyens de la transmission de toutes les nuances du skaz oral par la langue écrite. Il y montre comment l'auteur - Gogol - se cache sous le masque du narrateur et quels procédés il emploie pour styliser la langue orale aussi bien du narrateur que d'Akaky Akakievič, le héros de la nouvelle. Ce faisant, Eikhenbaum examine, en premier lieu, la structure des calembours et les procédés de l'influence sonore sur le lecteur-auditeur, en prêtant une attention particulière à l'aspect sonore-articulatoire de la parole et à la mimique. Pourtant, tout en montrant comment la parole d'Akaky Akakievič et d'autres personnages interagit avec la parole du narrateur, comment l'intonation change dans le texte et quel est son impact sur le lecteur-auditeur, Eikhenbaum ne recourt pas au concept de dialogue.

Cet article provoqua une vive réaction de plusieurs formalistes et devint un programme pour leur mouvement. Les questions de la spécificité du skaz en tant que genre littéraire, de son organisation, de l'image de l'auteur et du masque du narrateur, des rapports entre le langage oral et écrit ont été mise au centre des préoccupations des critiques littéraires en incitant aux recherches qui tâchaient d'y répondre.

Les articles de Vinogradov «Le sujet et la composition de la nouvelle Le Nez de Gogol» [Sjužet i kompozicija povesti Gogol'a « Nos »] (1921) et «De la morphologie du style naturel. L'analyse linguistique du poème pétersbourgeois Le sosie» [« $\mathrm{K}$ morfologii 
natural'nogo stil'a. Opyt lingvističeskogo analyse peterburgskoj poemy « Dvojnik »] (1922) font partie de ces recherches.

Dans le premier article, Vinogradov, tout comme Eikhenbaum, examine la composition de la nouvelle Le Nez et les procédés du jeu de mots. Tout comme Eikhenbaum, il analyse la nature des calembours de Gogol en remarquant qu'ils sont liés à la langue parlée. Mais, contrairement à Eikhenbaum, Vinogradov souligne que «les moments du jeu de mots apparaissent très en relief dans le dialogue. Cela aboutit à la parution de deux contextes, de deux plans de la parole qui se heurtent, se croisent, se superposent de multiples façons» (VINOGRADOV, 1976 [1921], p. 33), c'est-à-dire Vinogradov distingue le dialogue en tant qu'un des procédés dans la composition de l'œuvre littéraire qui permet de repérer le calembour le plus efficacement possible.

II faut noter que, en analysant le dialogue, Vinogradov, en premier lieu, le conflit, le choc, qui permet de jouer sur le double sens. Il va même jusqu'à la comparaison de deux rédactions de la nouvelle de Gogol et il montre comment Gogol a réécrit le dialogue pour éliminer les enchainements de mots qui empêchaient le double sens.

L'attention de Vinogradov porte également sur l'interaction de la parole de l'auteur avec la parole des personnages. II montre comment les mots de l'auteur investissent le skaz - la parole du narrateur - en le faisant se détourner de sa voie directe. Ces mots forment des ruptures logiques dans la narration.

A la fin de son article, Vinogradov arrive à la conclusion que c'est la langue parlée de tous les jours, «la langue extra-littéraire» d'où Gogol tire «une nouvelle logique des choses» qui lui sert de base de construction de son grotesque fantastique.

Ainsi, dans ce premier article, Vinogradov recourt déjà au dialogue en le considérant aussi bien comme un des procédés du jeu de mots que comme un des procédés de la composition.

Mais, dans le deuxième article cité (dans l'analyse linguistique du Sosie de Dostoïevski), une de ses tâches principales est de décrire les procédés de la construction des phrases du skaz et du dialogue ; de surcroît, il compare l'usage de ces procédés chez Dostoïevski et chez Gogol.

Dans Le Sosie, la narration passe à travers le masque d'un «humble narrateur des aventures», d'un fou Monsieur Goliadkine. Cela permet d'utiliser la même manière de décrire que celle utilisée par Gogol dans Le Nez et dans Les âmes mortes. 
La nouvelle Le Sosie est principalement caractérisée par un style narratif de la langue des affaires avec une nuance comique, construit sous l'influence directe du skaz de Gogol. Ce style interagit avec le style de la langue parlée d'un «pauvre petit fonctionnaire» Goliadkine. En outre, les deux styles sont investis par «de petits mots» et par «des expressions du registre populaire» de l'argot des fonctionnaires. Tout cela crée un enchevêtrement complexe des styles dans le tissus verbal de l'œuvre et fournit la clé à la compréhension de son architectonique.

Vinogradov analyse la parole de Goliadkine dans les menus détails en montrant comment se crée la caractéristique langagière du personnage. Ce faisant, il prête une attention particulière aux rapports entre les propos tenus par Goliadkine et ses mouvements, son expression motrice : ses regards, sa mimique, ses gestes.

A la fin de l'analyse des styles de la parole du narrateur et du héros, Vinogradov arrive à la conclusion que, à plusieurs reprises, Le Sosie se transforme en une forme du récit sur Goliadkine fait par son «sosie», c'est-à-dire par «un individu possédant sa manière de parler et ses notions» (VINOGRADOV, 1976 [1922], p.129). L'analyse de la parole de Goliadkine permet à Vinogradov d'établir les liens qu'elle entretient avec les procédés de la construction des « lettres d'ami» de Makar Dévouškine dans Les Pauvres gens.

Ainsi, dans ces articles, Vinogradov détermine sa sphère d'intérêts scientifiques l'analyse de l'œuvre de fiction - du skaz; la mise au jour de sa structure, des interactions entre l'auteur, le narrateur et les personnages à travers leurs styles de la parole. Ici même, Vinogradov touche à la question de la double fonction du dialogue: d'une part, c'est un procédé langagier de la transmission du style de la langue parlée qui permet de montrer les deux plans de la parole, mais, d'autre part, c'est aussi un des procédés de la construction de la composition. Ces deux fonctions du dialogue entrent en interaction au sein de l'oeuvre littéraire.

Dans son travail suivant, - son article «L'école du naturalisme sentimental. Le roman Les Pauvres gens de Dostoïevsky sur le fond de l'évolution littéraire des années 1840" [Roman Dostojevskogo « Bednyje ljudi » na fone literaturnoj evolucii 40-x godov] (1924), - les questions du sujet et de l'architectonique occupent une place de choix. Vinogradov considère Les Pauvres gens en tant qu'un roman bourgeois sentimental traditionnel - les échanges épistolaires entre deux amants. Les personnages principaux - un petit fonctionnaire pauvre, Makar Dévouškine, et l'orpheline, Varenka Dobroselova, s'écrivent 
pour se soutenir mutuellement dans la vie, d'où la tâche de Vinogradov d'analyser la forme du dialogue épistolaire. Cette forme a sa spécificité, parce que les lettres sont, au fond, des monologues. C'est pourquoi Vinogradov a besoin de déterminer le domaine d'activité des auteurs des lettres pour comprendre comment s'effectue la progression du récit. En outre, il avait besoin de comprendre comment la concaténation et l'enchevêtrement des lettres de Dévouškine et de Varenka s'unissaient afin de créer une structure monolithique. Comme le note Vinogradov, «ici, une nouvelle synthèse avait eu lieu, une synthèse qui a abouti à ce que les interventions littéraires de Dévouškine et les lettres de la pauvre orpheline deviennent les parties intégrantes d'une nouvelle composition littéraire unie» (VINOGRADOV, 1976 [1924], p. 176).

Depuis le début de l'analyse de ce roman, Vinogradov note que c'est Dévouškine qui joue le rôle principal dans cet échange épistolaire, alors que Varenka, dans la plupart des cas, ne joue que le «rôle du destinataire qui donne la réplique pour provoquer le changement des nuances du fond émotionnel dans les lettres de Dévouškine, pour lui faire faire telle ou telle action» (VINOGRADOV, 1976 [1924], p. 163), c'est-à-dire, il établit tout de suite les rôles des interlocuteurs dans ce dialogue.

Vinogradov prête constamment attention aux formes de la parole des personnages en s'y appuyant dans son analyse. II montre que, d'une part, Dévouškine écrivait en recourant à son style de fonctionnaire hérité des héros de Gogol, mais que, d'autre part, il était un des créateurs de nouvelles formes des œuvres littéraires. Quant à Varenka, elle est tout le contraire de Dévouškine: les thèmes traditionnels et la stylistique du sentimentalisme sont à la base de ses lettres.

Au cours de son analyse de l'architectonique du «dialogue» dans les lettres, Vinogradov distingue les lettres accentuées, qui représentent des points culminants de la narration et créent la dynamique du «spectacle» littéraire. En examinant le développement de la ligne dramatique, il souligne que, à la fin du roman, le dialogue se construit par le biais de l'enchevêtrement des formes expressives contrastées.

Ainsi, dans ce travail, Vinogradov étudie déjà le dialogue en tant qu'une des catégories principales de la composition de l'œuvre des belles-lettres pleine de dramatisme et de tension émotionnelle. Dans son analyse, Vinogradov montre comment Dostoïevski crée des rôles pour ses personnages, comment le changement de ces rôles conditionne la 
dynamique de l'action du roman, comment les lettres des héros entrent en interaction et comment cette interaction des «monologues» donne naissance à la tragédie.

On arrive donc à la conclusion que ce sont des questions générales de la création de la poétique et des méthodes objectives de l'analyse philologique des oeuvres des belleslettres qui préoccupaient Vinogradov dans la première moitié des années 1920. Son attention portait principalement sur l'étude de différentes formes de l'enchevêtrement des styles de la parole dans le tissus verbal de l'œuvre, sur son architectonique et sur la mise en évidence des rapports entre l'auteur, le narrateur et les personnages. Lors de l'analyse de ces questions dans l'œuvre de Gogol et de Dostoïevski, Vinogradov prenait en compte les fonctions des dialogues, mais il ne les considérait pas en tant qu'objets d'analyse à part entière. L'analyse du roman Les Pauvres gens de Dostoïevski - du dialogue épistolaire - a abouti à ce qu'il examine la question du dialogue en tant qu'une des formes principales de la composition de l'œuvre.

\section{Le dialogue dans la poésie}

En réalité, le dialogue en tant qu'un objet spécifique d'étude a été mis en avance par Vinogradov dans son analyse de la poésie. Cela s'est passé dans son travail «Sur la poésie d'Anna Axmatova » [O poezii Anny Axmatovoj] (1925) qui peut être considéré comme une réaction au livre de Boris Eikhenbaum «Anna Axmatova. (Un essai de I'analyse) » (1922). Si Eikhenbaum a pris pour but de montrer par le truchement des œuvres d'Axmatova les possibilités et les aspirations de la poésie moderne russe, Vinogradov poursuivait un autre but. Il a cherché à mettre en évidence la spécificité du style propre d'Axmatova, en passant par l'analyse des procédés langagiers de son système individuel et isolé. En même temps, Vinogradov a tendu encore vers un deuxième but, à savoir, démontrer la méthode d'analyse d'une œuvre littéraire établie par lui-même.

Dans l'analyse stylistique d'un œuvre littéraire, Vinogradov distingue deux composants : le symbolique et la composition. Dans son travail susmentionné, il met le symbolique artistique de la poétesse au centre de son étude. Cependant, il consacre le dernier chapitre de son travail critique à l'analyse de la composition des poèmes. Il l'intitule les «Grimaces du dialogue », en faisant ainsi une allusion au troisième chapitre du livre d'Eikhenbaum, dans lequel ce dernier démonte la nature articulatoire-mimique de la poésie 
d'Axmatova et place la mimique langagière même à la base de la composition des poèmes entiers ( EIKHENBAUM, 1969 [1922], pp. 120-130).

A la différence d'Eikhenbaum, Vinogravov voit l'approche aux poèmes d'Axmatova comme à un monologue - skaz dans lesquels s'introduit un dialogue. Une telle approche ouvre les nouvelles possibilités des effets émotionnelles qui sont déterminés par les interactions du dialogue avec le ton général de la narration et par les particularités de l'alternance des répliques.

En appuyant sur ces deux facteurs, Vinogradov distingue trois grands groupes des poèmes.

Le premier groupe est composé par les situations dans lesquelles un dialogue détermine la composition d'un poème tout en abaissant le skaz jusqu'au niveau de la description des détails d'une situation, autrement dit, jusqu'au niveau des remarques. Souvent, dans ce type des poèmes, on ne trouve pas l'alternance des répliques laconiques, mais plutôt un entrelacement compliqué des éléments des paroles monologale et dialogale. Comme indique Vinogradov, plusieurs plans langagiers et psychologiques se croisent dans les différentes directions. La poétesse peut se présenter à la fois comme une narratrice qui donne le fond des actions et comme une participante du dialogue, et avec cela sa réplique se développe jusqu'au monologue qui à son tour, inclut les fragments du dialogue. Comme un exemple de telle composition Vinogradov propose le poème «Je crispais mes mains sous le sombre châle... ». On peut aussi trouver un variant simplifié de cette composition qui est construit sur la base du dialogue dramatique, c'est-à-dire, sous la forme d'une « conversation » suivie par les remarques d'auteur.

A l'intérieure de ce groupe Vinogradov distingue encore deux sous-groupes: dans le premier, c'est la nature qui devient une «participante» de la conversation - «le chuchotement d'automne", "quelqu'un invisible dans l'ombre des arbres... "; dans le deuxième sous-groupe, ce sont les interlocuteurs inconnus au lecteur. Une question ou bien un monologue d'un interlocuteur provoque un discours de réponse. Avec cela, la situation générale reste énigmatique au lecteur. Et peu à peu, en appuyant seulement sur les remarques dans le monologue du héros ou bien de la héroïne principale, le lecteur peut la reconstruire. Vinogradov précise que dans ce groupe des poèmes le fond aperceptif reste indéfini. 
Dans le deuxième groupe, la base des poèmes est composée par skaz dans lequel sont introduits les mots d'autrui. Cela peut être une brève remarque de «lui » ou bien un longue monologue adressé à la héroïne. Dans ce dernier cas, la narratrice trace la situation et le personnage. Vinogradov met en relief ici un procédé qui est souvent utilisé par Axmatova : c'est le "cachetage du visage ", quand se croisent deux plans discursifs : le skaz de l'héroïne et la reproduction du monologue qui l'a troublé. Et avec cela, ces deux plans sont présentés dans un éclairage émotionnel "trompeur» pour le lecteur, tandis que le personnage, le discours de qui transmet la héroïne, reste énigmatique, bien qu'il a y les allusions à son apparence :

*«Elle est venu me torturer trois fois.

Je me suis réveillée dans un cri d'angoisse

Et j'ai vu ses bras minces,

Une bouche sombre, moqueuse.

«Avec qui échangeais-toi ce baiser,

A l'aube, devant les portes noires,

Quand tu jurais de mourir si

On vous séparait... »

Et c'est seulement dans le dernier appel à «l'interlocutrice » que sa nature se dévoile :

«Ce n'est pas en vaine que la tromperie a ri,

La tromperie, qui ne m'a pas été pardonnée $»^{2}$.

Vinogradov inclut aussi dans ce groupe les poèmes qui sont présentés sous la forme du skaz, mais qui ont une seule courte réplique comme la base de leur composition. II décrit différents rôles sémantiques lesquels peut jouer cette réplique dans la structure de composition : cela peut être les «mots du bien-aimé », cela peut être les expressions des objets d'art ou bien de la nature :

* «A l'arrière, la fumée qui s'épaissit,

\footnotetext{
${ }^{2}$ La traduction des poèmes et des citations marqués par * sont prises dans le livre Anna Axmatova. Requiem et autres poèmes. 1909-1963. Textes français présentation et notes par Henri Deluy. Tours, Ed. : Farrago, 1999, p.40. Les citations sans ce signe faites par l'auteur d'article.
} 
Le chant triste des violons : ... »

ou bien :

* «Les peupliers bruissent derrière la fenêtre :

«Ton roi n'est plus sur cette terre $»^{3}$.

Souvent, ce type de réplique dévoile l'image dans laquelle s'incarne l'héroïne : «II a plaisanté : « Ma funambule,

Comment tu survivras jusqu'en mai ? »

La réplique mise au début, peut créer une énigme : « Le garçon m'a dit :

«C'est si douloureux ».

Elle peut également servit d'un démêlement :

« Je viendrai et arrêterai sur le seuil,

Je dirai : «Rends à moi mon mouchoir »

Le troisième groupe est composé, selon Vinogradov, par les poèmes dans lesquels la place centrale est prise par un dialogue entrecoupé. Il est composé selon ses schémas particuliers et possède ses spécificités sémantiques. En décrivant tout cela, Vinogradov accorde une attention particulière aux cas dans lesquels une action se manifeste dans le dialogue même, c'est-à-dire, dans l'alternance des répliques. Dans ces cas, le skaz trace seulement le cadre. II prépare la perception du lecteur, en organisant son fond aperceptif. Dans tel situation, le dialogue commence par les phrases «stéréotypées" qui sont habituelles pour ce type de situation. Cependant, l'interlocuteur est caché. A tel type de début s'ajoute brusquement une réplique émotionnelle qui crée le contraste, la dissonance :

* «Elle partait, elle était près de la rampe,

Elle lui disait, dans un dernier effort :

«C'est tout... Ah, non, j'oubliais,

Je vous aime, je vous aimais déjà,

Même alors! »-

«Oui... » ${ }^{4}$

Ce procédé, c'est-à-dire, la construction de la réplique par la connexion des deux parties différentes qui donne en résultat une nouvelle formation émotionnelle - oxymoron,

\footnotetext{
${ }^{3}$ Ibid., 42

${ }^{4}$ Ibid., p.35.
} 
Vinogradov voit comme une des spécificités du dialogue chez Axmatova. Il décrit le moyen par lequel Axmatova, en utilisant le phénomène de l'aperception et des «phrases stéréotypées ", prépare la perception du lecteur et crée une tension émotionnelle qui est résolve par une nouvelle réplique agrégée par deux phrases stéréotypées propres à deux plans différents du dialogue.

Un autre procédé indiqué par Vinogradov est l'utilisation par Axmatova des stéréotypes langagiers du dialogue dans les situations contractées. L'effet de tel contraste est créé par la présence inhabituelle de ces répliques stéréotypées dans une situation tragique qui modifie complétement la perception des répliques.

« Dans la maison, ils se déplaçaient en silence, Ils n'attendaient plus rien.

Ils m'amenaient au malade

Et je ne l'ai pas reconnu ».

En contraste à cette situation tragique de la mort, le dialogue entre l'héroïne et son interlocuteur commence comme une conversation banale avant un départ en voyage.

« Il a dit : « Maintenant, Dieu merci », -

Et il est devenu absorbé par ses pensées. -

«Il a y longtemps que j'ai dû partir,

J'attendais seulement toi ».

Et puis, cette conversation banale se termine de façon inattendue par une réplique de la demande du pardon :

«Dis, peux-tu pardonner à moi?

Et j'ai répondu : « Je peux ».

$<\ldots \ldots . .>$

Soudain, la dernière force

Vivifie ses yeux bleus:

«C'est bien, que tu as pardonné à moi,

Tu n'étais pas toujours bénigne ".

En analysant ces procédés, Vinogradov arrive à la conclusion que la spécificité de la créativité d'Axmatova revient à « un système original du positionnement des morceaux de mosaïque » (VINOGRADOV, 1976 [1925], p. 457). 
Il trouve le même procédé dans l'alternance des répliques quand le lien du sens entre les brèves répliques soit est interrompu, soit devient logiquement contrasté, comme si les interlocuteurs regardent en profondeur de chacun, au-dessus des mots. La narratrice, quant à elle, décrit les manifestations externes des émotions qui accompagnent la conversation :

« Je l'ai rejoint au portail, j'ai crié :

«Mais ce n'était pas sérieux de ma part.

Je plaisantais. Je mourrai si tu pars. »

Son sourire alors, calme atrocement :

«Ne reste pas, m'a-t-il dit, par ce vent. »

Vinogradov propose plusieurs exemples de telle non-correspondance des répliques, autrement dit, de la stylisation de la conversation des gens comme s'ils ne s'entendent pas.

De plus, au cours de son analyse du dialogue, Vinogradov régulièrement souligne la façon comment Axmatova décrit la situation de la conversation donnée, quelles gestes et mouvements des héros elle met en relief :

«Et, en levant son main fin,

Il a légèrement touché les fleurs:

« Raconte-moi, comment on t'embrassée,

Raconte-moi, comment tu embrasses ».

De plus, Vinogradov précise que l'absence de telles indications est aussi significative et joue son rôle dans le sens général du poème.

Ainsi, on peut conclure que dans son analyse du dialogue chez Axmatova, Vinogradov s'appuie beaucoup sur la perception du lecteur, tout en utilisant les notions de perception et des phrases stéréotypées, ainsi que le phénomène de la naissance du nouveau sens produit par la juxtaposition de deux répliques. II prend en compte aussi bien les caractéristiques des interlocuteurs, que l'environnement dans lequel se déroule un dialogue. On résulte que Vinogradov fait son analyse en utilisant les principes d'organisation du dialogue décrits par Lev Jakubinskij dans son article "Sur la parole dialogale » (1923). Vinogradov non seulement se réfère à cet article au début de son chapitre, mais aussi montre comment on peut appliquer ces principes à l'analyse des textes poétiques. Par telle voie Vinogradov prouve deux thèses importantes : la première - que l'analyse de la parole poétique doit avoir pour base les découvertes et les donnes de la linguistique moderne et 
pas du tout une simple intuition de chercheur; la deuxième - que l'étude d'un œuvre littéraire doit prendre en compte les différents types de la composition de parole et leurs interactions.

\section{Le monologue et le dialogue en tant que constructions de la composition et de la parole}

On peut supposer que l'analyse du dialogue dans la poésie d'Anna Akhmatova ait servi à Vinogradov de base pratique pour le développement ultérieur de sa théorie et de sa méthode d'analyse de la langue de l'oeuvre littéraire qui furent formulées dans ses articles «Le problème du skaz dans la stylistique» [Problema skaza v stilistike] (1925, publié en 1926), «De la théorie des styles littéraires» [K teorii literaturnyx stilej] (1925, publié en 1927), et enfin dans son travail Sur la prose des belles-lettres [O xudožestvennoj proze](1930).

Vinogradov revient au problème du skaz, à son architectonique. Il critique, de manière cohérente, la position d'Eikhenbaum qui considère le skaz en tant que synonime de la «parole vivante», en tant qu'illusion de la parole, et démontre que le skaz - c'est, avant tout, une analogie littéraire de une des formes de la parole monologale.

Le skaz est une forme particulière et combinée du langage littéraire qu'on perçoit sur le fond des formations monologiques construites de même nature, propres à la pratique commune des interactions verbales. Le skaz est une construction littéraire élevée au carré, car il représente une superstructure esthétique placée au-dessus des constructions langagières (monologues) qui reflètent, eux-mêmes, les principes de la mise en forme compositionnelle et esthétique ainsi que les principes du choix stylistique» (VINOGRADOV, 1927, p. 28).

Une telle définition du skaz incite à définir les constructions langagières à proprement parler, le monologue et le dialogue. C'est pourquoi, en s'appuyant sur les travaux d'E. Budde (1859-1929), de L. Ščerba, de L. Jakubinsky, Vinogradov compare le monologue et le dialogue dans la parole. Il partage leur opinion selon laquelle le dialogue prime dans les échanges verbaux dans la société, c'est pourquoi le dialogue est d'un grand intérêt pour les linguistes. Mais, quant au monologue, Vinogradov se trouve en désaccord Ščerba pour qui le monologue représente une forme conservatrice de la parole qui reflète la norme de la langue. Vinogradov souligne que le monologue est une forme particulière de la construction stylistique dans la parole qui, «tout en restant dans les limites du lexique et du 
sytème grammatical d'un parler donné, crée des parallèles stylistiques, forge la phraséologie, détermine les fonctions stylistiques de divers schémas syntaxiques. La parfaite maîtrise des formes de la parole monologale est un art...» (VINOGRADOV, 1927, p. 30). Vinogradov note que les linguistes n'ont pas encore commencé à étudier la parole monologale, ainsi, il ne donne pas le classement des formes de cette parole, il se concentre sur le domaine de la parole quotidienne. Dans ce domaine, il distingue quatre types du monologue: le monologue persuasif - forme primitive du discours de l'orateur, le monologue lyrique - forme de l'expression des émotions, le monologue dramatique - un type complexe de la parole «dans lequel la langue des mots n'est qu'une sorte d'accompagnement d'autres systèmes des phénomènes psychiques - par le biais du langage de la mimique, des gestes, des mouvements plastiques», et enfin le monologue du type référentiel ${ }^{5}$. Pour distinguer ces formes du monologue, Vinogradov ne recourt pas au principe thématique, c'est la distinction des fonctions du langage qui est à la base de sa typologie: la fonction persuasive du langage, le langage affectif, le langage référentiel.

II note que c'est le monologue dramatique qui est le plus proche du dialogue, parce qu'il se caractérise par le lien des unités phrastiques avec des messages gestuels et mimiques, avec les mouvements du corps: «le monologue dramatique est, au fond, une forme d'un dialogue tendu, avec des répliques omises. Il se construit selon les principes de la parole dialogique et représente une sorte de concaténation des répliques séparées» (VINOGRADOV, 1927, p. 31).

En ce qui concerne le monologue référentiel, il s'appuie sur la structure logicoobjective de la langue et se divise en deux sous-types: le monologue-raisonnement, en tant que forme primitive du «langage scientifique», et le monologue-narration. C'est ce dernier sous-type qui est lié à la notion du skaz dans la littérature et c'est ce monologue que Vinogradov analyse en détail pour démontrer les rapports complexes entre la parole monologique et le genre du skaz.

Ainsi, en concentrant ses efforts sur l'analyse du monologue dans la parole et sur sa transformation - le skaz - dans la littérature, Vinogradov ne perd jamais de vue le dialogue en comparant ces deux catégories de la composition.

Cette comparaison se développe également dans son travail Sur la prose des belleslettres (1930) où le niveau des généralisations théoriques est plus élevé. En définissant la

\footnotetext{
${ }^{5}$ Ibid.
} 
nature du langage de l'auteur, Vinogradov parle de deux contextes de l'œuvre littéraire: du contexte des formes esthético-littéraires de la parole et du contexte des systèmes sociolangagiers qui coexistent dans les limites de la langue écrite des livres et du «parler de l'intelligentsia» d'une époque donnée. Par conséquent, l'étude de la langue de l'œuvre des belles-lettres doit être aussi bien sociolinguistique que stylistico-littéraire.

Selon Vinogradov, dans le mécanisme de la transformation des formes langagières dans l'œuvre de belles-lettres, les catégories de la composition jouent un rôle important, car elles changent du point de vue historique et adaptent leur structure à la typologie des genres littéraires. En conséquence, ces catégories doivent être étudiées sur le fond des formes de la composition de la langue écrite et de la langue parlée. En attachant une telle importance aux formes de la composition, Vinogradov consacre un chapitre entier à leur description qu'il intitule "Des catégories de la composition et de la parole dans la littérature». C'est là où il compare les lois de la construction du monologue et du dialogue au sein de l'oeuvre littéraire. II parle de la nécessité d'élaborer la conception des types compositionnels de la parole dans le domaine de la création littéraire et considère une telle conception en tant qu'une des directions principales des études de la langue des belleslettres. Dans le chapitre ci-dessus, il argumente et formule les problèmes principaux de cette direction des recherches. II note, en particulier, que «la compréhension des raisons de la coexistence de différentes formes de la parole dans les constructions monologiques, la compréhension des principes selons lesquels elles intègrent le dialogue - c'est un nouveau problème important des études de la langue des oeuvres littéraires» (VINOGRADOV, 1980 [1930], p. 78).

Plus loin, Vinogradov trace les voies principales de l'étude de ce problème en faisant des observations intéressantes sur la spécificité du dialogue. Ainsi, il note que la parole dialogale fait non seulement partie du tissus verbal de différents types de la poésie et de la prose, mais aussi qu'elle organise elle-même des genres littéraires indépendants (Vinogradov pense ici aux oeuvres dramatiques). Par conséquent, les fonctions de la parole dialogale, les moyens de sa construction et la sémantique de ses éléments peuvent différer radicalement d'un genre à l'autre. Par exemple, dans la prose narrative, on trouve souvent «le dialogue monologuisé», alors que la «dialoguisation» des monologues, - qui se laissent percevoir comme soliloque du personnage ou comme sa conversation avec un interlocuteur absent, - domine dans le drame. En ce qui concerne la nouvelle, ici, selon Vinogradov, il y a 
plus de possibilités pour le dialogue unilatéral, parce qu'une autre série de répliques peut exister potentiellement, sans jamais se réaliser (il cite l'exemple du roman de J. Webster Papa-Longues-Jambes). II mentionne également les formes «écrites» du dialogue, c'est-àdire les formes qui ne se projettent pas dans la parole et qui peuvent être utilisées pour la composition de la nouvelle ou du récit (par exemple, Un roman en neuf lettres de Dostoïevski ou La correspondance de Tourgueniev).

Vinogradov analyse à part le dialogue dans le drame en formulant tout un bloc des questions et des buts de la recherche. Parmi eux, on peut nommer la «discontinuité» psychologique du dialogue qui crée la possibilité de comprendre les répliques sur plusieurs plans: en tant qu'«expressions» des images des personnages aussi bien que formes de leur représentation par l'auteur; en tant que moyens de la délimitation (thématique, expressive, sociale) précise de la parole des personnages, en tant que dépendance de la sémantique du dialogue des facteurs extra verbaux, en tant que forme de la concaténation des parties dialogiques qui se caractérisent par de diverses dépendances de la vie courante et du sujet, en tant que questions liées aux types de l'interaction de l'expression verbale avec l'expression gestuelle et mimique, etc. Tout cela a permis à Vinogradov de parler de la nécessité d'analyser à part la sémantique et la syntactique du dialogue dramatique.

En outre, Vinogradov n'oublie pas de noter le lien du problème du dialogue dramatique avec la théorie de la parole scénique, c'est-à-dire avec les questions de la réalisation théâtrale du texte. II fait une observation importante de ce que le moment «théâtral» est également compris dans les formes de la construction de la parole dans le dialogue.

Il est intéressant de noter que Vinogradov avait l'intention de s'occuper de ces problèmes du langage du drame lui-même. Les recherches du célèbre philologue soviétique A.P. Čudakov (élève de Vinogradov), effectuées dans les archives, en portent le témoignage ${ }^{6}$. Ainsi, en 1926-1927, lors de son travail dans l'Institut d'Etat de l'histoire des arts (GIII), Vinogradov s'occupait déjà du thème « le problème du langage du drame». On a des informations sur ses multiples conférences au sujet de cette question données pendant

\footnotetext{
${ }^{6}$ Čudakov, A. P. «V.V. Vinogradov i teorija xudožestvennoj reči», in Vinogradov. O jazyke xudožestvennoj prozy.

Izbrannye trudy. Moskva : Nauka, pp. 285-315, 1980. ['Vinogradov et la théorie du langage des œuvres littéraires'].
} 
ces années. De surcroît, comme le note Čudakov, dans les lettres de Vinogradov de la fin de l'année 1927, il est fait mention de ce qu'il écrit un livre sur le dialogue, plus précisément, sur le langage dramatique (il existe des titres différents de ce livre: Le langage du drame, La théorie du dialogue). Mais, comme le pense Čudakov, après avoir commencé à recueillir des matériaux pour le livre L'évolution du naturalisme russe, Vinogradov abandonna ce projet.

Ainsi, vers l'année 1930, la théorie de la langue des oeuvres des belles-lettres se forme dans l'esprit de Vinogradov. Dans cette théorie, l'étude de la spécificité du monologue et du dialogue, perçus comme différents systèmes de la composition langagière, occupe une des places principales.

\section{Le dialogue dans l'œuvre rhétorique}

Si la première partie de l'ouvrage Sur la prose des belles-lettres est consacrée aux œuvres littéraires (à la nouvelle, au récit, au roman), la deuxième partie de ce livre est dédiée à la question de la rhétorique vue comme théorie normative qui détermine les genres de la prose et les principes selon lesquels ils se construisent. Vinogradov note que le but principal de la rhétorique est d'examiner les formes de la construction de l'œuvre littéraire selon les lois du lecteur, c'est-à-dire selon les principes de la construction sur lesquels se base la «suggestion», la «persuasion» de l'auditeur-interlocuteur. Vinogradov cite les travaux des linguistes russes et européens, tels que Vinokur, Svedelius, Bally, Špitzer, relatifs aux problème de l'auditeur et aux formes des interactions sociolangagières. Il analyse à part la conception de la parole rhétorique du philosophe russe Gustav Špet (1879-1937) en établissant ses liens avec la parole pragmatique qui a une orientation expressive et tâche de faire impression. En acceptant, dans les grandes lignes, le point de vue de Špet, Vinogradov lui objecte sa pensée de la nature rhétorique de tout roman et parle, pour sa part, de l'interaction des procédés rhétoriques et poétiques.

Comme exemples d'analyse, Vinogradov retient trois oeuvres où il s'agit d'un seul et même évènement - «l'affaire Kronenberg» au sujet de la violence exercée par le banquier sur sa fille de sept ans: le discours de V. Spasovič, avocat de Kronenberg, et les réactions qu'il provoque auprès des écrivains Dostoïevski et Saltykov-Ščédrine. Lors de cette analyse, Vinogradov prend obligatoirement en compte le facteur de la composition. 
Ainsi, il considère le discours de l'orateur en tant qu'une forme particulière du monologue dramatique «adapté à l'atmosphère du «spectacle» social de tous les jours ou du «spectacle» civil» (VINOGRADOV, 1980 [1930], p. 120). Tout est important dans ce spectacle: le principe de la «théâtralisation», l'atmosphère générale, le «jeu» de l'avocat en tant qu'«acteur», c'est-à-dire ses poses, ses mouvements, sa mimique, ses gestes et, en outre, la «mélodie» intérieure de la parole qui s'exprime à travers le rythme et le système des changements des intonations. Pourtant, ce domaine lié à l'art de la performance de l'orateur, domaine «du théâtre social», constitue, pour Vinogradov, une discipline à part l'oratoire, distincte de la rhétorique dans le sens propre du terme. L'oratoire a partie liée avec la science des formes de la construction littéraire des œuvres rhétoriques. C'est ce point de vue des belles-lettres qu'adopte Vinogradov pour analyser le discours de Spasovič. Il établit quelques principes d'organisation de l'œuvre rhétorique en notant, en particulier, l'importance du dialogue dont la structure est déterminée par la fonction d'«exemple», «d'illustration»: «le dialogue n'est pas le discours direct des personnages sur eux-mêmes, c'est une forme de leur représentation sous l'angle des jugements moraux de la part du rhéteur» (VINOGRADOV, 1980 [1930], p. 146).

Plus loin, Vinogradov passe à l'analyse du discours de Spasovič donnée par Dostoïevski dans son Journal de l'écrivain. C'est là où Vinogradov montre comment Dostoïevski dévoile les procédés rhétoriques de Spasovič tout en introduisant d'autres formes de l'art oratoire dans son œuvre. L'écrivain transfère la question de la sphère juridique dans le domaine social, commun à tous les hommes: «L'âme de l'enfant devant la cour pénale». C'est un oxymore, thème contradictoire dans son essence même, qui détermine la dynamique des séries de signification chez Dostoïevski.

Vinogradov commente en détail l'analyse de Dostoïevski en montrant que cette analyse se construit, au fond, comme «dialogue» de l'écrivain avec l'avocat, entrecoupé par la «conversation» avec les auditeurs. Cette manière de s'adresser, à tour de rôle, tantôt à Spasovič, tantôt aux auditeurs - le procédé de changement des «plans» expressifs - crée une tension dramatique. Dostoïevski utilise également le monologue dramatique basé sur les citations du discours de Spasovič, mais il le transforme en discussion. Vinogradov appelle ce procédé «l'illusion littéraire du dialogue», car Spasovič ne riposte pas. C'est comme si Dostoïevski lisait à Spasovič des bribes de son discours, mais en modifiant complétement leurs intonations, leurs formes expressives. C'est pourquoi, le discours de Spasovič se 
déforme lors d'une telle transmission «comme une citation dans un contexte hostile» (VINOGRADOV, 1980 [1930], p. 161).

A la fin de son analyse de la réaction de Dostoïevski, Vinogradov arrive à des conclusions intéressantes sur la spécificité du dialogue dans l'oeuvre rhétorique. II note que la «duplicité» intérieure, immanente au dialogue, constitue sa caractéristique importante: dans la structure verbale de l'oeuvre rhétorique, il y a deux images - celle de l'auteur et celle de son «adversaire». De surcroît, il faut opérer la différence entre une telle «double nature» du sujet et l'auditeur ou le lecteur dont on veut emporter l'adhésion et qui est plutôt un allié. Par conséquent, Vinogradov parle de l'impossibilité d'établir des rapports analogiques entre un tel dialogue et le dialogue dans la langue quotidienne qui contient deux sujets socialement présents: l'auteur et son interlocuteur. Vinogradov considère le monologue de l'acteur dans le drame comme analogue à un tel dialogue rhétorique, parce que, dans le drame, le monologue s'adresse au spectateur, mais, de par sa construction, il vise un autre partenaire. Seulement, à la différence du drame, le «partenaire» dans l'œuvre rhétorique se conçoit toujours comme adversaire, comme image négative autour de laquelle se crée l'atmosphère expressive correspondante. En conséquence, Vinogradov considère le monologue rhétorique en tant que forme de la structure dialogique «polyphonique». II arrive à la conclusion qu'«il en résulte non pas le dialogue des personnes, mais celui des conceptions du monde qui s'incarnent conventionnellement dans des personnes» ((VINOGRADOV, 1980 [1930], p. 167).

Ainsi, en utilisant les catégories de l'approche communicative, Vinogradov était le premier dans la linguistique et la critique littéraire russes à distinguer un type particulier du dialogue qui représente une des structures de l'organisation de l'oeuvre rhétorique. En le considérant en tant que «dialogue des conceptions du monde», Vinogradov trace les voies des études ultérieures de ce dialogue.

\section{Le dialogue dans la Dame de pique de Pouchkine}

Si dans les années 1920, dans le processus de la définition d'un objet de la stylistique, Vinogradov étudiait les œuvres de Dostoïevski et de Gogol, dans le début des années 1930, il a concentré son attention sur la prose de Pouchkine. Aussi bien sa théorie du langage des œuvres littéraires, achevée et publiée à cette époque, que se méthodologie d'analyse 
peuvent être appliquée au matériel concret, d'autant plus que, selon une remarque de Vinogradov, pour ce moment-là, le style de Pouchkine et surtout sa prose n'ont pas été analysés.

Vinogradov a pensé que la manière de narration de Pouchkine avait bien muri dans la «Dame de pique » qui est caractérisée par un système des différents plans qui se trouvent en mouvement et qui se croissent, ce qui en résultat crée un sens multiple et riche de cet œuvre. La composition complexe, elle aussi, joue un rôle important, car elle inclut l'entrelacement de la narration et de la parole dialogale.

Comme lors de l'analyse de la poésie d'Axmatova, Vinogradov concentre son attention sur cet aspect, en partant de la thèse que l'organisation du dialogue dépend de la structure de narration. Pour cette raison, il analyse en détail les rapports entre le contexte monologique de l'auteur ou du narrateur et les conversations des personnages, tout en mettant son analyse dans un chapitre à part « Dialogues dans la composition de la « Dame de pique $"$.

Vinogradov considère la dramatisation de l'action comme une des fonctions principales du dialogue. Il explique par cela le fait que dans le premier chapitre, la narration est remplacée par une représentation presque scénique de la conversation commune des joueurs des cartes. On peut voir que la narration là-bas inclut, en effet, les « remarque d'un mettre-en scène ", et que la fonction du narrateur est donnée à Tomskij. Cependant, dans le dialogue, ce dernier ne montre pas sa personnalité littéraire du narrateur, mais plutôt son caractère mondain du joueur et d'homme du monde. Ainsi se passe le dédoublement personnel du style de narration.

Vinogradov indique encore un procédé intéressant : déjà dans le récit de Tomskij, où se trouve un croissement des différents plans personnels, Tomskij reproduit les discours des personnages de son récit dans les mêmes formes lexiques et syntaxiques dans lesquelles elles ont été produit par ces personnages eux-mêmes. Cependant, il le fait avec une expression ironique, avec un «accent » du narrateur qui les transmet. Par exemple, en rencontrant sur sa grande mère, la vieille comtesse, il transmet son discours, en ajoutant I'ironie : « - elle pensait lui faire honte en lui démontrant avec condescendance qu'il a y dette 
et dette, et qu'il existe une différence entre un prince et un carrossier. Bernique! Grand-père regimbait... ${ }^{7} \ldots$

Dans son analyse Vinogradov aussi prend en compte le caractère de l'alternance des répliques et indique que dans les remarques d'auteur marquant cet alternance les verbes sont utilisé au passé perfectif, afin de montrer le changement des mouvements et des états d'esprit des personnages: « demanda le maître de la maison», «dit l'un des convives», «les joueurs redoublèrent d'attention». II tire la conclusion que ce procédé syntaxique détermine le dynamisme du dialogue qui est une essence de l'action dramatique.

Dans le deuxième chapitre de la nouvelle, plus exactement, dans la scène à la maison de la comtesse, la position du narrateur change : il prend le rôle de l'observateur qui décrit et saisi le sens des événements et du monde. Ce changement influence aussi le dialogue dramatique qui se brise en fragments (en éclats, selon les termes de Vinogradov) commentés par le narrateur: le dialogue est remplacé par une simple information du narrateur.

Dans ces cas, I'auteur non seulement décrit les mouvements qui accompagnent le dialogue, mais de plus, il explique leur sens, c'est-à-dire, met le dialogue au niveau de la citation narrative qui a besoin à son tour les commentaires. On peut dire que le dialogue est dissout dans la narration. Ainsi, dans telle situation, les scènes dramatiques ne avancent pas la nouvelle, comme dans le premier chapitre, mais eux-mêmes sont entrainées avancées par la narration. Formellement, cela est exprimé par la jonction des parties narratives aux répliques par la conjonction «i ». Le dialogue ainsi devient un maillot syntaxique de la narration : «- Morte ! dit-elle, et moi qui ne le savais même pas! Nous avons été nommées ensemble demoiselles d'honneur, et lorsque nous nous présentâmes, I'Impératrice... Et pour la centième fois, la comtesse raconta l'anecdote à son petit-fils »"

De plus, Vinogradov montre comment toutes les scènes dramatiques du deuxième chapitre se transfèrent de façon symbolique dans la conscience de Lizavèta Ivanovna, c'està-dire, dans sa parole interne. Cela souligne encore plus la fonction narrative du dialogue :

\footnotetext{
${ }^{7}$ La citation est prise chez Vinogradov, 1980 [1936], p 205. La traduction est prise dans le livre A. Pouchkine. La Dame de pique. Traduction du russe Michel Niqueux. Ed. Librairie du Globe, Paris, 1999, p.27.

${ }^{8}$ La citation est prise chez Vinogradov, 1980 [1936] : 206. La traduction est prise dans le livre A. Pouchkine. La Dame de pique, traduction du russe Michel Niqueux. Ed. Librairie du Globe, Paris, 1999, p.43.
} 
«Et voilà mon existence! » songea Lizavèta Ivanovna» ${ }^{9}$. Vinogradov considère ce procédé comme une des spécificités typiques du dialogue chez Pouchkine

Outre la dissolution du dialogue dans la narration, Vinogradov met en évidence une autre spécificité du style de Pouchkine - c'est la dramatisation "pluri subjective» [raznosubjektnnaja] de la narration elle-même, lorsque un monologue d'auteur ou bien du narrateur se transforme en «polylogue ». Dans tel monologue d'auteur, les différentes voix commencent à résonner ce qui crée un entrelacement des différentes lignes personnels et des différents points de vue. Vinogradov voit cela comme une innovation de Pouchkine laquelle a été développé plus tard dans la prose de Dostoïevski et de Saltykov-Ščedrine.

Comme encore un autre procédé novateur de Pouchkine, Vinogradov indique la différenciation socio-caractéristique du langage des personnages. Ce procédé apparait à partir du deuxième chapitre de la "Dame de pique ». Dans la littérature russe avant Pouchkine, régnait le solipsisme sentimental du personnage ce qui excluait la diversité des caractères sociaux. Pouchkine donc commence à utiliser le principe de l'individualisation typique du caractère qui se manifeste dans sa manière de parler et qui correspond à la diversité réelle des variantes sociales. En analysant les styles de parole de Lizaveta Ivanovna, d'Hermann et de la comtesse, Vinogradov définit leurs traits typiques et leur rôle dans la création des images psychologiques des personnages. II affirme que dans la construction des dialogues se heurtent les différents systèmes du langage qui, en leur tour, se différent du style de narration. Cependant, le monologue d'auteur ne détruit pas des traits caractéristiques individuels du langage des personnages.

Résultant son analyse, Vinogradov affirme que Pouchkine transforme le dialogue prosaïque et que grâce à ces transformations le style du dialogue devient réaliste. De plus, Vinogradov relève encore un aspect important du dialogue chez Pouchkine - ce sont les procédés originaux de la combinaison du langage avec la mimique, les gestes, les postures et les mouvements. II les considère comme les nouvelles formes de la motricité figurative chez Pouchkine : les postures, la mimique, les gestes des personnages décrits dans cette nouvelles ne contiennent le pathos déclamatif. Tous ces mouvements sont liés aux émotions et aux états d'esprit d'un héros de façon "métonymique ", c'est-à-dire, ils se trouvent dans les rapports diverses, «figuratifs " avec une situation et avec un rôle du personnage. Par exemple, dans certaines scènes, Pouchkine utilise le principe de non-

\footnotetext{
${ }^{9}$ Ibid., p.51.
}

Eutomia, Recife, 21(1): 20-47, Jul. 2018 
correspondance entre le langage et les mouvements. On peut le voir dans la scène de la conversation de Lizavèta Ivanovna et de mademoiselle qui lui a apporté un billet d'Hermann. Ce contraste entre les mots et les émotions, les mots et les mouvements est un des procédés typiques de la dramatisation chez Pouchkine.

Ainsi, en concluant ces observations des rapports entre la narration et la parole dialogale dans la «Dame de pique », Vinogradov affirme que

les scènes dialogiques frappantes par son laconisme interviennent dans la narration dans les situations les plus dramatiques, parfois pour commencer, parfois pour terminer, en tout cas, comme si elles cherchent à concentrer le sens essentiel de la scène. Et avec cela, la simplicité, la nature réaliste et la fragmentation augmentent leur tension émotionnelle. (VINOGRADOV, 1980 [1936], p. 219).

De plus, il indique que «sans l'analyse du dialogue chez Pouchkine, il est difficile à comprendre la réforme de la manière de la description littéraire-langagière des personnages qui a été utilisée dans les années 1830. Cette réforme a préparé Gogol avec sa création des grimaces langagiers et des grotesques » (Ibid., p. 215).

On peut conclure, que dans son analyse du dialogue chez Pouchkine, Vinogradov trace la ligne du développement de la littérature russe et montre la filiation des idées et des procédés littéraires. Sa méthode d'analyse lui a permis de démontrer le « polylogue » chez Pouchkine et par cela a établi les liens entre la prose de Pouchkine et celui de Dostoïevski.

Ainsi, on voit que dans les années 1930, Vinogradov continue à développer son analyse du dialogue. Aussi bien dans ses travaux théoriques que dans l'analyse pratique des œuvres littéraires, il met en relief le dialogue en tant qu'un objet d'étude en le liant, en général, avec l'organisation de la composition des œuvres de fiction et avec l'interaction des différents styles langagiers qui représentent les points de vue de l'auteur, du narrateur et des personnages. Dans ce domaine, les intérêts et les positions de Vinogradov se croissaient avec le livre de M. Bakhtin «Problèmes de la création de Dostoïevski » publié en 1929.

\section{Conclusion}

En analysant la conception du dialogue chez Vinogradov, nous arrivons aux conclusions suivantes: 
1. Le problème du dialogue attira l'attention de Vinogradov dès le début de son activité scientifique, c'est-à-dire, à partir des années 1920. La discussion sur la nature du skaz menée par les formalistes russes a mis devant eux la question de la correspondance entre la parole quotidienne et son imitation dans un œuvre littéraire. Au cours de cette discussion Vinogradov a proposé sa propre définition du skaz qui se différait de celui-ci d'Eikhenbaum.

Si Eikhenbaum affirmait que à la base de la prose se trouve un skaz en tant qu'une imitation de la parole orale, Vinogradov lui a objecté en prouvant que le skaz est seulement la représentation littéraire d'une des formes de la parole orale monologale, c'est-à-dire, qu'il faut prendre en compte sa transformation esthétique dans une œuvre fictionnelle. Pour cette raison, il est plus juste à parler plutôt de la distinction des formes et des fonctions de I'utilisation de la parole orale en fiction, que de voire en skaz une simple imitation. En défendant sa conception, Vinogradov indiquait à mainte reprise que même si les constructions langagières de la parole des œuvres littéraires sont formées et reçus sur le fond des constructions stylistiques existantes dans le langage ordinaire, elles ne doivent pas être identifiées à ces dernières. En conformité avec cela, le dialogue de la parole quotidienne sert seulement une base de l'analyse du dialogue dans un œuvre littéraire.

Au cours de la formation de sa théorie de l'œuvre littéraire, Vinogradov a relevé les fonctions du dialogue suivantes: en tant que le langage des personnages, il est un des moyens de la constitution de l'image psychologique d'un personnage, en tant qu'un élément important de la composition, il sert le moyen principale de la dramatisation; en tant qu'un structure principale de composition, il peut, lui-même, organiser certaines genres littéraires indépendants, comme par exemple, le drame.

2. En participant activement dans les discussions des années 1920 sur les tâches de la stylistique, Vinogradov reliait ce domaine de la philologie à la linguistique. Pour cette raison, dans son analyse de la parole dialogale, il s'appuyait sur les recherches modernes des linguistes russes et occidentaux. En Russie, c'était le travail de Lev Jakubinskij «Sue la parole dialogale ", dans lequel à la première fois en linguistique, ont été définis les paramètres de l'organisation du dialogue. Des auteurs occidentaux, Vinogradov mentionnait les travaux de Leo Špitzer, de Van Guinneken, d'Os. Laurié. Il est fort possible, que c'est grâce à son maître A. Šaxmatov (1864-1920) qui avait introduit la notion de «communication» dans la linguistique russe, Vinogradov se réfère au travail de Carl Svedelius, ainsi que les recherches de Ch. Bally et de $\mathrm{H}$. Wünderlich. Dans ces travaux il 
emprunte l'idée de l'«auditeur » vers qui est orientée la communication et qui influence I'organisation de la parole. Ainsi, dans I'analyse du dialogue, Vinogradov s'appuyait toujours sur l'interaction entre la critique littéraire et la linguistique.

3. Vinogradov analyse le dialogue dans les différents genres de littérature - la poésie, la prose de fiction, les œuvres de la rhétorique. Si on peut voir les problèmes de l'intégration du dialogue dans la narration, son entrelacement avec le monologue d'auteur ou d'un narrateur, ses fonctions dans la structure verbale d'œuvre littéraire comme un des points généraux d'étude de Vinogradov, dans chaque analyse concrète, il utilise les paramètres particuliers pour montrer la spécificité du style d'écrivain.

Dans les dialogues d'Axmatova, il souligne le rôle du fond aperceptif qui prépare la perception du lecteur. Vinogradov montre les différents procédés utilisés par la poétesse - la « masque » d'héroïne, l'image de son interlocuteur, l'usage des répliques stéréotypées et à la fin, d'une réplique - réaction inattendue qui modifié complètement le sens du poème tous cela pour créer le sens dramatique des poèmes. Ces procédés Vinogradov considère comme la spécificité du style d'Axmatova.

Dans son analyse de la «Dame de pique » de Pouchkine, Vinogradov se fonde sur la différenciation socio-historique du langage des personnages qui contribue à la constitution de la caractéristique psychologique de leurs images. II analyse les différents rapports entre le dialogue et la situation, entre la parole et les actions des personnages et montre les innovations de Pouchkine dans ce domaine. Ici, Vinogradov parle non de la confrontation des caractères de personnages, comme dans les dialogues d'Axmatova, mais d'entrecroisement des plans subjectifs entiers qui représentent les différents points de vue.

Dans son analyse du dialogue d'un œuvre rhétorique, Vinogradov s'appuie sur les paramètres de l'organisation de la parole en prenant en compte la situation et la réaction des auditeurs, en plus, ce dernier paramètre inclut non seulement les auditeurs neutres, mais aussi l'auditeur - adversaire. Pour ce type du dialogue Vinogradov propose un terme spécial de "l'illusion littéraire du dialogue », car il ne contient pas des répliques de la réponse réelles. Vinogradov souligne que la spécificité la plus importante de ce dialogue est le fait que c'est un dialogue de la conception de vie.

4. Ainsi, on peut dire chez Vinogradov, dialogue est un des composants les plus importants de sa théorie de l'œuvre littéraire. Il est analyse en lien et en comparaison avec le monologue. Et avec cela Vinogradov fixe son attention particulière sur l'interaction de ces 
deux catégories de la composition verbale. Telle approche lui a permis d'établir un bloque des problèmes d'étude du monologue et du dialogue en tant que les principes de la construction des œuvres littéraires et a démontré le rôle joué par dialogue dans définition du style spécifique de chaque écrivain.

\section{Références bibliographiques}

ALPATOV, V. Vološinov, Bakhtine i lingvistika. Moskva, "Jazyki slavianskix kultur ». 2005. [Vološinov, Bakhtine et la linguistique]

AXMATOVA, A. Requiem et autres poèmes. 1909-1963. Textes français présentation et notes par Henri Deluy. Tours, Ed. : Farrago, 1999.

BOLŠAKOVA, A. Theorija avtora u M. Bakhtina i V. Vinogradova (na materiale russkoj « derevenskoj prozy ». In: Dialogue. Carnaval. Xronotop, N², pp. 4-22, 1999.

[Théorie d'auteur chez Bakhtine et Vinogradov (sur le matériel de la «prose de la campagne »]

ČUDAKOV, A. V. V. Vinogradov i teorija xudožestvennoj reči. In: O jazyke xudožestvennoj prozy. Izbrannyje trudy. Moskva : Nauka, pp.285-315, 1980.

[Vinogradov et la théorie du langage des œuvres littéraires]

EIKHENBAUM, B. Illuzia skaza. In: Skvoz' literaturu, Leningrad, p.151-164, [1918] 1924.

[L'illusion du conte]

. Kak sdelana « Šinel' » Gogolja. Poètika, pp.151-165, 1919.

[Comment fait le « Manteau » de Gogol]

Anna Axmatova. Opyt analiza. In: recueil: O poèzii. Leningrad, Ed: Sovetskij pisatel', pp. 75-147, [1922] 1963.

[Anna Axmatova. Essaie de l'analyse]

JAKUBINSKIJ, L. O dialogičeskoj reči. In :Russkaja reč', pp. 96-194, 1923.

[Sur la parole dialogale]

PERLINA, N. Zabavnyje slučai slučajutsja na puti k bakhtinskomu forumu. In : Dialogue. Carnaval. Xrohotop. $\mathrm{N}^{\circ} 1$, pp. 20-37, 1995 a.

[Les situations amusantes se produisent en route vers le colloque sur Bakhtin]

Dialog o dialoge: Bakhtine - Vinogradov, 1924-1965. In: Bakhtinologija. Issledovanija, perevody, publikacii. Saint-Pétersbourg: Alteja, pp.155-170, 1995b.

[Dialogue sur dialogue : Bakhtine-Vinogradov, 1924-1965] 
POUCHKINE, A. La Dame de pique. Traduction du russe Michel Niqueux. Paris. Ed. : Librairie du Globe, 1999.

VINOGRADOV, V. Sjužet i kompozicija povesti Gogolja « Nos ». In: VINOGRADOV, V. Poètika russkoj literatury. Izbrannyje trudy. Moskva : Nauka, pp.5-44, [1921] 1976.

[Le sujet et la composition de la nouvelle le « Nez » de Gogol]

K morfologii natural'nogo stil'a. Opyt lingvističeskogo analyse peterburgskoj poemy "Dvojnik». In: VINOGRADOV, V. Poètika russkoj literatury. Izbrannyje trudy. Moskva: Nauka, pp.101-140, [1922] 1976.

[De la morphologie du style naturel. L'analyse linguistique du poème pétersbourgeois Le sosie]

Roman Dostojevskogo «Bednyje ljudi » na fone literaturnoj evolucii 40-x godov. In : VINOGRADOV, V. Poètika russkoj literatury. Izbrannyje trudy. Moskva: Nauka, pp.141-187, [1924] 1976.

[L'école du naturalisme sentimental. Le roman Les Pauvres gens de Dostoïevsky sur le fond de l'évolution littéraire des années 1840]

. O poezii Anny Axmatovoj. In: VINOGRADOV V. Poètika russkoj literatury. Izbrannyje trudy. Moskva : Nauka, pp. 369-459, [1926] 1976.

[Sur la poésie d'Anna Axmatova]

Problema skaza v stilistike. In : Poètika, Leningrad, Académia, v.1, pp.24-40, 1926.

[Les problèmes du skaz en stylistique]

O xudožestvennoj proze. In: VINOGRADOV, V. O jazyke xudožestvennoj prozy. Izbrannyje trudy. Moskva : Nauka, pp. -175, [1930] 1980.

[Sur la prose fictionnelle]

Styl «Pikovoj damy». In: VINOGRADOV. V. O jazyke xudožestvennoj prozy. Izbrannyje trudy. Moskva: Nauka, pp.176-239, [1936] 1980.

[Style de la «Dame de pique »]

\footnotetext{
i Ivanova, Irina, ancienne maître de conférences à I'Université de Saint-Pétersbourg (Russie) et maître d'enseignement et de recherches à l'Université de Lausanne, section des langues slaves. Domaines des recherches: I'histoire des idées linguistiques russes, la circulation des idées entre la Russie et l'Occident, la culture russe au début du $X X^{\mathrm{e}}$ siècle, la didactique des langues étrangères.

Dans l'histoire des idées linguistiques, les points d'intérêt sont les suivants : la constitution de la phonétique expérimentale aussi bien en France qu'en Russie ; les rapports entre la langue et la pensée, entre la langue et la musique ; la naissance de la théorie de dialogue et d'étude de la parole.

irinaivanova@yahoo.com
} 